\title{
O Trabalho no SUS: retrato das relações trabalhistas no Programa Academia da Saúde
}

\section{The labor at SUS: portrait of labor relations in the Academia da Saúde Program}

\author{
DOI: $10.46919 / \operatorname{arch} 2$ n5-007
}

Recebimento dos originais: 01/05/2021

Aceitação para publicação: 31/06/2021

\section{Fernanda Ramos Parreira}

Doutora em Sociologia (UFG). Professora Adjunta da Faculdade de Educação Física e Dança (FEFD/UFG). Professora colaboradora no Mestrado Profissional em Saúde Coletiva (IPTSP/UFG). Goiânia (GO), Brasil. Endereço: Av. Esperança, s/n - Chácaras de Recreio Samambaia, Goiânia - GO, E-mail: fernandarparreira@ufg.br

\section{Marta Rovery de Souza}

Doutora em Ciências Sociais (UNICAMP). Professora Titular no Departamento de Saúde Coletiva (IPTSP/UFG) Goiânia (GO), Brasil. Endereço: R. 235, S/n - Setor Leste Universitário, Goiânia - GO, E-mail: martaroverysouza@ufg.br

\section{RESUMO}

Justificativa: As contratações na área da saúde pública brasileira - especialmente em nível municipal perpassam por essas novas dinâmicas laborais. Trabalhadores da saúde contratados por tempo determinado, relações trabalhistas sem estabilidade ou garantias mínimas de direitos trabalhistas e previdenciários. Objetivo: Refletir sobre as dinâmicas laborais existentes no contexto de um programa. Método: Trata-se de um estudo descritivo-transversal, que utiliza dados secundários referente ao programa Academia da Saúde, bem como dados oriundos de pesquisa de campo. Resultados: Predomínio de profissionais do sexo feminino, jovens, com elevado grau de instrução, presença de equipes multiprofissionais, mas com relações e vínculos de trabalho precarizados, constituídos por meio de contratações temporárias, por exemplo credenciamento, baixa permanência de trabalhadores. Conclusões: As relações de trabalho - no contexto do programa Academia da Saúde, no estado de Goiás - constroem-se de forma precarizada e sem estabilidade ao trabalhador, e contribui a pouca motivação e desinteresse de profissionais em atuarem no programa.

Palavras-chave: Gestão do Trabalho no SUS, Trabalhadores da Saúde, Subempregabilidade, Atenção Primária à Saúde

\begin{abstract}
Background: Brazilian public health hiring - especially at the municipal level - permeates these new work dynamics. Health workers on a fixed-term basis, unsettled labor relations or minimum guarantees of labor and social security rights. Objective of paper: Reflect on the existing work dynamics in the context of a program. Method: This is a descriptive cross-sectional study that uses secondary data related to the Academia da Saúde program, as well as data from field research. Results: Predominance of female, young, highly educated professionals, presence of multiprofessional teams, although presenting precarious working relationships and bonds, constituted through temporary hiring, for example, accreditation, low permanence of workers. Conclusions: Labor relations - in the context of the Academia da Saúde program
\end{abstract}


in the state of Goiás - are built in a precarious manner and without stability to the worker, contributing to the professionals' lack of motivation and lack of interest in working in the program.

Keywords: Work Management in SUS, Health Workers, Underemployment, Primary Health Care

\section{INTRODUÇÃO}

A precarização do trabalho e seus impactos na vida dos trabalhadores, especialmente no Brasil, têm sido tema recorrente em estudos e pesquisas, uma vez que nas últimas décadas observa-se um aumento significativo de relações laborais galgadas no processo de terceirização, "pejotização", contratos por credenciamento e informalidade laboral.

Em Goiás, conforme dados disponibilizados pelo Instituto Mauro Borges de Estatística e Demografia, o setor público apresentou nos anos de 2016 e 2017 um déficit de admissões de servidores públicos, conforme tabela 1 .

Tabela 1 - Flutuação do nível de ęmprego por atividade eçonômica, no estado de Goiás, 2016 e 2017.

\begin{tabular}{|c|c|c|c|c|c|c|}
\hline \multirow{2}{*}{ Atividade econômica } & \multicolumn{3}{|c|}{2016} & \multicolumn{3}{|c|}{2017} \\
\hline & Admissão & Desligamento & Saldo & Admissão & Desligamento & Saldo \\
\hline Total & 557.337 & 576.691 & -19.354 & 577.658 & 552.288 & 25.370 \\
\hline Extrativa mineral & 1.732 & 2.608 & -876 & 1.752 & 1.912 & -160 \\
\hline Indústria de transformação & 97.263 & 104.076 & -6.813 & 99.655 & 94.870 & 4.785 \\
\hline Serv. ind. utilidade pública & 2.156 & 2.099 & 57 & 3.021 & 3.435 & -414 \\
\hline Construção civil & 58.412 & 62.643 & -4.231 & 55.384 & 53.706 & 1.678 \\
\hline Comércio & 130.976 & 138.803 & -7.827 & 136.869 & 131.339 & 5.530 \\
\hline Serviços & 199.723 & 202.695 & -2.972 & 208.718 & 197.890 & 10.828 \\
\hline Administração pública & 422 & 460 & -38 & 244 & 384 & -140 \\
\hline Agricultura, silvicultura etc. & 66.653 & 63.307 & 3.346 & 72.015 & 68.752 & 3.263 \\
\hline
\end{tabular}

O presente estudo tem por objetivo refletir sobre as dinâmicas laborais existentes no contexto de um programa ministerial - Programa Academia da Saúde - e os possíveis impactos/ ou o que isso gera que essas relações geram na sustentabilidade e consolidação das políticas públicas.

O trabalho, a princípio, constitui-se uma necessidade para manter o metabolismo social entre humanidade e a natureza. Após o processo de industrialização e urbanização o trabalho serviu ao processo de alienação do trabalhador (ANTUNES, 1999; 2005 e ANTUNES \& PRAUN, 2015). Logo o trabalho tornou-se um potencial produtor de sofrimento e de adoecimento. Deste modo, as condições de trabalho impactam nas condições de saúde e vida do indivíduo e do coletivo. "[A] forma de inserção dos homens, 
mulheres nos espaços de trabalho contribui decisivamente para formas específicas de adoecer e morrer" (BRASIL, 2002).

A dupla dimensão do trabalho é um tanto contraditória e dicotômica, pois a passo que cria, emancipa, humaniza e produz; também escraviza, precariza, desumaniza, reproduz e aliena (ANTUNES, 2005). Para além das questões estruturais presentes no campo do trabalho, no tocante ao universo da gestão pública, as relações de trabalho são de considerável relevância para a sustentabilidade e consolidação de políticas públicas.

As mudanças socioeconômicas de cunho neoliberal e globalizante exigiam, no contexto da gestão pública brasileira, uma nova configuração na administração pública, baseado no modelo gerencial (BRESSER-PEREIRA, 2010).

As mudanças na estrutura estatal trazem novas conformações inclusive nas relações de trabalho, pois através da transferência da gestão a organismos não-governamentais e/ou organizações sociais, bem como a flexibilização na contratação de servidores a partir dessa nova lógica de gestão pública. Esse cenário impacta substancialmente na área da saúde, e o novo modelo de gestão pública se torna presente no cerne da saúde pública e direcionada a essa "nova ordem" de globalização e neoliberal e contrapondo-se as premissas propostas pelo SUS e defendida pelo Movimento de Reforma Sanitária (MRS) (ROCHA \& SOUZA, 2011).

Observa-se que as relações trabalhistas perpassam por processos de flexibilização das contratações através da pejotização e terceirização. Para Rocha \& Souza (2011), no serviço público, especialmente na saúde, esse processo de flexibilização ocorre com a instituição de medidas que oportunizam a contratação do tipo temporária, bancos de horas, voluntariado, e outras condutas que visam a redução dos custos com a força de trabalho.

É importante mencionar, como aponta Mariano (2017), que a partir de 2016, o Brasil assevera o processo de construção de políticas voltadas a adoção de medidas severas de austeridade fiscal, que impactam de forma substantiva nos investimentos para o fomento de serviços essenciais e políticas sociais, cita-se a emenda constitucional n. 95/2016 como marco dessa mudança social, econômica e política. Em 2017, institui-se a reforma trabalhista, sob os moldes apregoados pelo modelo de austeridade, intensifica o processo de precarização das relações de trabalho (RODRIGUES \& MELO, 2017).

\section{MÉTODOS E TÉCNICAS}

Trata-se de um estudo observacional descritivo, que utiliza dados secundários referente ao programa Academia da Saúde. Os dados foram extraídos do sistema formSUS, formulário eletrônico do SUS, referente ao Ciclo Anual de Monitoramento, no período de 2015 a 2017, realizado com os municípios habilitados junto ao programa $(n=125)$, no estado de Goiás, disponibilizados pela Secretaria de Estado da 
Saúde do Estado de Goiás. Deste modo, considera-se também como estudo longitudinal retrospectivo. As variáveis analisadas são quantitativas (discreta) e qualitativas (nominal e ordinal): tipo de profissional que atua nos polos; tipo de vínculo empregatício; avaliação do programa, e dificuldades dos municípios habilitados no programa em relação a gestão de pessoas.

Ademais, recorreu-se a pesquisa de campo. Quatro municípios goianos participaram dessa etapa do estudo, sendo realizada entrevista, do tipo semi-estruturada, com 05 (cinco) profissionais vinculados ao Programa Academia da Saúde e 11 (onze) usuários. A seleção dos entrevistados foi realizada através da técnica metodológica de bola-de-neve (snowball), junto aos polos do programa.

Tendo em vista que o presente artigo é derivado de pesquisa de doutoramento, vale destacar que a pesquisa foi submetida e aprovada pelo Comitê de Ética, da Universidade Federal de Goiás (CAAE: 64921517.3.0000.5083). Ademais, em virtude de parte do estudo necessitar de dados e documentos disponibilizados pela Secretaria de Estado da Saúde do Estado de Goiás (SES-GO), também foi aprovado pelo Comitê de Ética Local da Superintendência de Educação em Saúde e Trabalho (SES-GO).

\section{RESULTADOS}

O programa Academia da Saúde foi instituído no âmbito do Sistema Único de Saúde por meio de portaria ministerial, n. 719 de 07 de abril de 2011, no intento de tornar concreta a implementação da Política Nacional de Promoção da Saúde (CONASS, 2011).

O panorama nacional, através de monitoramento do programa Academia da Saúde, em 2017, retrata que $98,6 \%$ dos municípios respondentes $(n=1.372)$ oferecem práticas corporais e atividades físicas, $81,9 \%$ ações com enfoque na temática de alimentação saudável e adequada, 79,2\% realizam atividades de educação em saúde, $23,5 \%$ promovem práticas artísticas e culturais e apenas 13,4\% enfocam em atividades vinculadas as práticas integrativas e complementares.

Em 2017, 98,7\% dos polos respondentes do monitoramento anual, realizado através de formulário eletrônico - FormSUS ( $\mathrm{n}=75)$, em Goiás, informaram desenvolver ações focadas nas práticas corporais/atividade física, $92 \%$ desenvolvem práticas de educação em saúde e $84 \%$ atividades de promoção de alimentação saudável.

É importante destacar, que diferentemente do estado de Goiás que apresentam maior atuação de fisioterapeutas, no contexto nacional do programa $(n=1372)$, conforme relatório de monitoramento do Ministério da Saúde (Brasil, 2017), 90,5\% dos profissionais que atuam no programa Academia da Saúde são profissionais de Educação Física, seguido pelo profissional fisioterapeuta (55\%); nutricionista (54,1\%), psicólogo (42,2\%) e assistente social (32,6\%). Deste modo, diversos artigos publicados com enfoque no perfil do profissional que atua no PAS apresentam o perfil predominante acima apresentado, a exemplo Guarda et. al. (2015). 
Enquanto que no estado de Goiás, no mesmo período, 85,1\% dos polos em funcionamento possuíam fisioterapeutas em seu quadro profissional, seguido por profissionais de educação física $(75,7 \%)$, nutricionistas $(70,3 \%)$ e psicólogos $(56,3 \%)$, conforme apurado pela pesquisadora através dos dados disponibilizados pela Secretaria de Estado da Saúde.

Os profissionais entrevistados são predominantemente do sexo feminino, jovens (faixa de idade entre 20 a 49 anos), apresentam um grau de formação importante, possuindo pós-graduação em nível de especialização. Especializações relacionadas ao campo de sua formação inicial como ortopedia, terapias manuais, neurologia, saúde da família, acupuntura e fisiologia do exercício. As profissionais entrevistadas ressaltam a importância do trabalho multiprofissional e interdisciplinar no programa.

Acerca do vínculo laboral, substancialmente, os municípios optam por contratações temporárias, como o credenciamento. No caso do Brasil, conforme dados extraídos do ciclo de monitoramento 2017 e apresentados em Tabela 2.

TABELA 2 - Tipo de vínculo empregatício presentes nos polos em funcionamento, Brasil - 2017.

\begin{tabular}{ccccccc}
\hline & \multicolumn{2}{c}{ Número de Polos Respondentes } & \multicolumn{3}{c}{ Percentual } \\
\hline Tipo de Vínculo & Sim & Não & Total & Sim & Não & Total \\
\hline Estatutário (concursado) & 829 & 835 & 1664 & 49,8 & 50,2 & 100 \\
\hline Temporário & 1139 & 525 & 1664 & 68,4 & 31,6 & 100 \\
\hline Celetista (CLT) & 222 & 1442 & 1664 & 13,3 & 86,7 & 100 \\
\hline Cedido de outro órgão & 233 & 1431 & 1664 & 14,0 & 86,0 & 100 \\
\hline Estagiário & 199 & 1465 & 1664 & 12,0 & 88,0 & 100 \\
\hline Comissionado & 185 & 1479 & 1664 & 11,1 & 88,9 & 100 \\
\hline Voluntário & 177 & 1487 & 1664 & 10,6 & 89,4 & 100 \\
\hline
\end{tabular}

Fonte: elaborado pelas autoras com base em dados do Ciclo de Monitoramento Anual do PAS 2017

Em Goiás a mesma realidade se apresenta quanto à flexibilização das relações trabalhistas no SUS, especialmente no Programa Academia da Saúde como é possível observar na Tabela 3.

TABELA 3 - Tipo de vínculo empregatício presentes nos polos em funcionamento, Goiás - 2017.

\begin{tabular}{ccccccc}
\hline & Número de Polos Respondentes & \multicolumn{3}{c}{ Percentual } \\
\hline Tipo de Vínculo & Sim & Não & Total & Sim & Não & Total \\
\hline Estatutário (concursado) & 31 & 43 & 74 & 41,9 & 58,1 & 100 \\
\hline Temporário & 67 & 7 & 74 & 90,5 & 9,5 & 100 \\
\hline Celetista (CLT) & 3 & 71 & 74 & 4,1 & 95,9 & 100 \\
\hline Cedido de outro órgão & 10 & 64 & 74 & 13,5 & 86,5 & 100 \\
\hline Estagiário & 5 & 69 & 74 & 6,8 & 93,2 & 100 \\
\hline Comissionado & 8 & 66 & 74 & 10,8 & 89,2 & 100 \\
\hline Voluntário & 10 & 64 & 74 & 13,5 & 86,5 & 100 \\
\hline
\end{tabular}

Fonte: elaborado pelas autoras com base em dados do Ciclo de Monitoramento Anual do PAS 2017 
Permite, inclusive, afirmar que o processo de flexibilização e precarização dos vínculos trabalhistas se apresentam mais intensivo no território goiano em relação ao programa Academia da Saúde ao se comparar as tabelas 2 e 3 .

As relações trabalhistas são tão sensíveis no contexto do programa Academia da Saúde que na modalidade efetivo/concursado, em Goiás, que nos anos de 2015 era de 37,7\%, 31,7\% em 2016 e em 2017 eram de $41,9 \%$. Sendo este tipo de vínculo à segunda forma mais utilizada pela gestão municipal para captação de recursos humanos para o programa. E essa condição de instabilidade empregatícia retrata o tempo de manutenção dessas profissionais que gira em torno de 05 anos e 06 meses, levantada na etapa de visita in loco aos 04 municípios pesquisados. E junto ao programa Academia da Saúde por volta de 02 anos e 06 meses.

Acerca da contrapartida municipal, exigida na portaria de adesão ao PAS, em 2015 54,3\% dos municípios participantes afirmaram que a contratação de profissionais é uma ação de contrapartida empreendida pelo município. E esse percentual aumenta substancialmente no ano de 2016 para 87,3\% e $83,8 \%$ no ano subsequente (ano 2017).

Essa instabilidade dos vínculos trabalhistas gera impactos à gestão, pois de um lado dificultam a continuidade do serviço, e do outro gera insegurança ao profissional tanto em relação aos seus direitos trabalhistas, quanto em relação a sua permanência após o término de seu contrato, presente, por exemplo, na fala a seguir:

[...] se fosse assim, olhar pro contrato, eu não tenho direito a férias, não tenho direito a $13^{\circ}$, eu não tenho direito a entregar um atestado, então eu acho assim, que é amor, sabe, amor aos meus pacientes, e tudo... [...] Eu pensei que eu ficaria só um ano. De verdade. Eu pensei que eu ficaria um ano, mas já foram dois anos e três meses (risos) (PROFISSIONAL A).

[...] igual nesse contrato nosso, a gente não tem direito, né, a $13^{\circ}$, nem férias, nem nada, isso desmotiva, desmotiva o servidor pra trabalhar. Só que no meu caso, no caso da, da outra colega, ela, é o mesmo contrato, a gente não tem direito a nada, nem atestado pode trazer, né? Então assim, é complicado, mas mesmo assim a gente não cruza os braços, né? A gente quer oferecer porque a gente ama o que faz e acolhe a comunidade. A comunidade depende do nosso trabalho, né? Então assim, o município tem que fazer a sua parte? Tem que fazer, mas nós também temos que mostrar, né, o serviço e honrar ali, a sua profissão (PROFISSIONAL B)

Outro ponto que cabe ressaltar, é que essa multiplicidade de formas de contratação e captação de recursos humanos na gestão pública acende outro problema relacionado às relações interpessoais entre profissionais com vínculos distintos. Pois, para Nogueira et. al. (2004), as múltiplas formas de gestão de contratos mobilizaram mudanças na lógica de gestão interna dos órgãos públicos e geraram conflitos diversos, pelo tratamento desigual conferido a cada ente contratado. 
Essa percepção de tratamento desigual se apresenta no discurso das profissionais entrevistadas, especialmente entre os trabalhadores com vínculo provido por concurso público e aqueles com cargos de livre nomeação e exoneração, o caso dos cargos em comissão e contratos por tempo determinado.

\begin{abstract}
Aí tem até uma certa rixa assim, as pessoas pensam que... os contratados pensam que os concursados têm alguma vantagem, e os concursados sempre acham que os contratados é que têm, porque eles, né, têm aquela... mas assim, eu graças a Deus, eu entrei em uma gestão diferente, essa gestão foi mudada, e aí a gente tenta deixar bem claro que a gente só quer trabalhar (PROFISSIONAL D).
\end{abstract}

Outro impacto é a quebra de confiança estabelecida entre o profissional e usuário. Pois com a alternância de profissionais a confiança e o vínculo entre profissional e usuário sofrem mudanças negativas. Como avalia o Usuário 4 seu relacionamento com a profissional do programa.

[...] sem querer expor, mas, eh, no tempo da [nome suprimido] ela promovia muito mais animação, mais alegria... assim, sabe? [...] (USUÁRIO 4).

[...] Não vou dizer que é ruim não, mas ela que se precise de se abrir mais um pouquinho com a gente (USUÁRIO 4).

O processo de flexibilização das relações de trabalho no SUS geram a precarização do trabalho, a desvalorização do trabalhador e o comprometimento na qualidade do serviço prestado a sociedade (MARTINS, 2008; EBERHARDT, 2021 e MACHADO et.al., 2011). Assim, apontam à necessidade de repensar a gestão do trabalho no SUS. Pois, a gestão do trabalho no SUS não envolve apenas o gestor, mas todos os atores que participam na construção e consolidação do sistema de saúde, já que existe uma relação entre "[...] usuários, trabalhadores de saúde e gestores no processo de produção e consumo de cuidados de saúde" (CONASS, 2011).

\title{
4 DISCUSSÃO
}

Os profissionais de saúde vinculados ao programa Academia da Saúde são aqueles que atuam com as temáticas prioritárias em PS - práticas corporais/ atividade física e alimentação saudável e adequada. Destacam-se, assim, as categorias de fisioterapia; educação física e nutrição. Evidencia-se que os tipos de profissionais contratados coadunam as principais atividades desenvolvidas no polo do programa Academia da Saúde, práticas corporais/atividade física; educação em saúde; promoção de alimentação saudável.

$\mathrm{O}$ aspecto multiprofissional encontrado durante a pesquisa de campo demonstra a diversidade profissional presente no contexto do SUS, especialmente na atenção primária. Os trabalhadores do SUS são considerados os profissionais que atuam em diversos níveis da gestão pública e níveis da atenção à saúde que compõem direto ou indiretamente o Sistema Único de Saúde (MACHADO et. al., 2015). 
A ampliação do quadro de profissionais reconhecidos como do campo da saúde advém da transição paradigmática que compreende que o modelo biomédico é um modelo limitado e que não corresponde a todas as demandas de saúde, especialmente às psicossociais. Cardoso \& Hennington (2011) destaca que a composição de equipe multiprofissional é outorgada pela complexidade e pelas múltiplas dimensões que envolvem o campo da saúde - social, cultural, psicológica, ambiental e biológica. E tendo em vista o processo de especialização do conhecimento torna necessária à articulação de diversos campos do saber e profissionais.

Acerca da flexibilização dos vínculos trabalhistas no SUS destaca-se que esse processo se intensifica ao final da década de 1980, ganhando força no início na década de 1990. Apontam que essa tendência de desregulamentação e flexibilização surge ancorada no cenário de crise econômica nesse período (MACHADO et.al., 2015; NOGUEIRA \& RODRIGUES, 2004 e ASSUNÇÃO, 2011).

A intensificação desse processo que acaba por criar vínculos trabalhistas precários no sistema de saúde, no início do século XXI, faz com que o Ministério da Saúde se manifeste com preocupação e propõe um Programa Nacional de Desprecarização do Trabalho no SUS, o DesprecarizaSUS. No entanto, como se pode perceber com a realidade do programa Academia da Saúde a saída encontrada pelo Ministério da Saúde quanto a desprecarização do trabalho no SUS não se mostra efetiva nos territórios brasileiros e goianos, conforme dados apresentados.

O tipo de contratação desses profissionais não oferece estabilidade aos trabalhadores que atuam no referido programa, bem como impacta na sustentabilidade das ações e atividades desenvolvidas no PAS, uma vez que, a rotatividade de profissionais junto ao programa compromete substancialmente a consolidação e a efetividade do programa no território que se encontra implantado.

\section{CONSIDERAÇÕES FINAIS}

As incoerências e paradoxos encontrados aqui junto ao programa Academia da Saúde podem se dar possivelmente pelo fato deste se constituir um programa ancorado em uma nova proposta e concepção de saúde, focada no modelo ampliado de saúde. Não obstante, em diversos momentos é transversalizado pelo modelo tradicional de saúde, biomédico. Logo, observa-se uma subvalorização de categorias de profissionais de saúde que são distintas daquels de formação médica-hospitalocêntrica.

Mesmo com iniciativas como o DesprecarizaSUS, as contratações de profissionais por meio de credenciamento, terceirização ou como pessoa jurídica tem sido a forma adotada pela gestão municipal como forma de "desburocratizar" e garantir a continuidade de prestação de serviços. Vale acrescentar que tal discurso de flexibilização das relações trabalhistas é referendado pela reforma trabalhista, em Lei $n$. 13.467, de 13 de julho de 2017. 
Os dados revelam que as relações de trabalho - no contexto do programa Academia da Saúde, no estado de Goiás - constroem-se de forma precarizada e sem estabilidade ao trabalhador, e contribui a pouca motivação e interesse de profissionais em atuarem no programa.

Por fim, a precarização das relações de trabalho é um fator importante e que impacta substancialmente à condição de manutenção e continuação do programa e de políticas públicas com foco a promoção da saúde, assim como, ao enfraquecimento do próprio Sistema Único de Saúde.

\section{REFERÊNCIAS}

ANTUNES, R. Os Sentidos Do Trabalho: Ensaio sobre a afirmação e a negação do trabalho. São Paulo: Boitempo, 1999.

. O Caracol e sua Concha: Ensaios sobre a nova morfologia do trabalho. São Paulo: Boitempo, 2005.

ANTUNES, R.; PRAUN, L. A sociedade dos adoecimentos no trabalho. Serv. Soc. Soc [internet]. 2015 [acesso em 2021 jan 02] n. 123: 407-427, jul./set. Disponível em: https://doi.org/10.1590/0101-6628.030

ASSUNÇÃO, A. A (Org.). Trabalhar na saúde: experiências cotidianas e desafios para a gestão do trabalho e do emprego. Rio de Janeiro: Editora Fiocruz, 2011.

BRASIL. Ministério da Saúde. Secretaria de Políticas de Saúde. Departamento de Atenção Básica. Cadernos de Atenção Básica [CAB] n. 5 - Saúde do Trabalhador. Brasília: Ministério da Saúde, 2002.

BRASIL. Ministério da Saúde. Secretaria de Vigilância em Saúde. Departamento de Vigilância de Doenças e Agravos não Transmissíveis e Promoção da Saúde. Panorama nacional de implementação do Programa Academia da Saúde: monitoramento nacional da gestão do Programa Academia da Saúde: ciclo 2016 [recurso eletrônico]. Brasília: Ministério da Saúde, 2017.

BRESSER-PEREIRA, L. C. Democracia, Estado Social e Reforma Gerencial. Revista de Administração de Empresas, São Paulo, V. 30, N.1, p.112-116, 2010.

CARDOSO, C. G.; HENNINGTON, E. A. Trabalho em equipe e reuniões multiprofissionais de saúde: uma construção à espera pelos sujeitos da mudança. In: Fundação Oswaldo Cruz. Trabalho, Educação E Saúde. Fiocruz: Escola Politécnica De Saúde Joaquim Venâncio, - V. 09, Supl. 1, 2011.

CONSELHO NACIONAL DE SECRETÁRIOS DE SAÚDE. A Gestão do Trabalho e da Educação na Saúde / Conselho Nacional De Secretários De Saúde. Brasília: Conass, 2011.

GUARDA, F. R. B.; SILVA, R. N.; FEITOSA, W. M. N.; SANTOS NETO, P. M.; ARAUJO JUNIOR, J. L. A. C. Caracterização das equipes do programa academia da saúde e do seu processo de trabalho. Revista Brasileira de Atividade Física e Saúde [internet]. 2015. [acesso em 2021 jan 02] 20(6): 638-649. Disponível em: https://doi.org/10.12820/rbafs.v.20n6p638

MARIANO, C. M. Emenda constitucional 95/2016 e o teto dos gastos públicos: Brasil de volta ao estado de exceção econômico e ao capitalismo do desastre. Rev. Investig. Const. [internet]. 2017 [acesso em 2021 jan 02] 4(1) Curitiba Jan./Apr. Disponível em: https://doi.org/10.5380/rinc.v4i1.50289 
ROCHA, L. M.; SOUZA, M. A. S. L. A Condição Contemporânea Do Trabalho No Setor Público De Saúde: Uma Análise Da Desregulamentação Do Trabalho Nesse Setor A Partir Das Imposições Do Grande Capital. V Jornada Internacional De Políticas Públicas. Maranhão, 2011.

RODRIGUES, I. S.; MELO, J.C.D. Reforma Trabalhista: precarização das relações de trabalho diante da perspectiva instaurada pelas novas regras celetistas através da lei 13.467/2017. ViannaSapiens [internet]. 2017 [acesso em 2021 jan 02] 8(2), jul/dez. 288-310. Disponível em: https://doi.org/10.31994/rvs.v8i2.243

MARTINS, J. V. Interfaces Do Lazer: Educação, Trabalho e Urbanização. São Paulo: Casa do Novo Autor Editora, 2008.

EBERHARDT, L. D.; CARVALHO, M.; MUROFUSE, N. T. Vínculos de trabalho no setor saúde: o cenário da precarização na macrorregião Oeste do Paraná. Saúde em Debate [internet]. Rio de Janeiro, 2015. . [acesso em 2021 jan 02] 39(104): 18-29, jan.-mar. Disponível em: https://doi.org/10.1590/0103110420151040432

MACHADO, M. H.; VIEIRA, A. L. S.; OLIVEIRA, E. Gestão, trabalho e educação em saúde: perspectivas teórico-metodológicas. In: BAPTISTA, T. W. F. (Org.). Políticas, Planejamento e Gestão em Saúde: abordagens e métodos de pesquisa. Rio de Janeiro: Editora Fiocruz, 2015.

NOGUEIRA, R. P.; BARALDI, S.; RODRIGUES, V. A. Limites críticos das noções de precariedade e desprecarização do trabalho na Administração Pública. Observatório de Recursos Humanos em Saúde ObservaRH. Universidade de Brasília. Brasília: UnB, 2004.[acesso em 2019 abr 18] Disponível em: http://www.observarh.org.br/nesp 